\title{
PREMIUM CALCULATION WITHOUT ARBITRAGE Author's Reply on the Note by P. Albrecht
}

\author{
By G. VENTER
}

\begin{abstract}
Albrecht's interesting discussion raises two major points: is an arbitrage free insurance market a reasonable assumption, and are adjusted distribution principles appropriate?

I had assumed an arbitrage free insurance market without really discussing reasons in support of it. Certainly there have been arbitrage profits made through reinsurance, possibly, for example, by selling excess on excess at rates based on regular excess policies, and perhaps in the London Market Excess arena. But even if arbitrage occurs frequently, I feel that an arbitrage free market is still a reasonable assumption for theoretical developments, if we take it to mean that systematic ongoing arbitrage is not possible. Although some arbitrage situations have probably lasted a few years, all I am aware of eventually have disappeared as they became better known. Either entrepreneurs try to outbid each other to take advantage of them, or the sellers lose their willingness to play. A list of insurers that are regularly making arbitrage profits would be an interesting refutation of this assumption.
\end{abstract}

Albrecht gives some examples of the well known and quite true proposition that larger portfolios are more stable. Large insurers can maintain the same security level at a lower price. Our risk theory training leads actuaries to believe that the smaller needed security premium for large insurers will induce them to charge lower prices. This is not necessarily true in the market, however. Larger insurers may in fact charge the market price and make more profits, for example. Also, it would be fairly surprising if small insurers actually do get away with charging more for the same risks. If they do, they should buy prorata reinsurance heavily from the large insurers at the large insurers' risk price and pocket the difference. There are of course transaction costs to buying prorata reinsurance; those may in fact be the limit of what the smaller insurers can demand as the supposed bonus price they get from the market for being small.

Albrecht's discussion of adjusted distribution principles is an extension of the arguments I was making. Adjusted distribution principles, which include covariance rules like CAPM and its generalizations, constitute the class of additive premium principles. Albrecht's comment that requiring an additive principle eliminates only some of the inadequate premium principles, namely the non-linear ones, is all I was really trying to say. Most of the principles historically advanced have been non-linear, and just excluding those was enough for this paper, which was meant to be elementary.

Certainly there is more to be done to find specific additive principles that will work in the market for a particular line of insurance. For instance, principles 
that give prices below expected values would not be used in normal circumstances, nor would those whose loadings do not grow as fast as market prices do for higher layers. Additive principles can do either or both of those, so are too wide a class to use indiscriminately. Also some, like CAPM, have measurement problems that currently preclude their use in individual risk pricing. It is hard enough to measure the covariance for a line of insurance, let alone for layers or risks. There is an empirical component to finding working principles, but more developed theory such as the martingale and options approaches Albrecht outlines should help narrow the search.

Lacking a properly supported and practically applicable theory, actuaries use ad hoc methods. In this context simple approaches like adjusting the parameters of their distributions bear trying. I was surprised by the example that shows that a scale transform can lead to a price below the expected cost for a strange enough insurance product. Due to its simplicity, the scale transform is a good place to start. However, judging by market costs, it probably does not lead to high enough loadings for upper layers. From the example given, it certaintly should not be used to price a coverage for the retained portion below a franchise deductible. If a principle works well in the market for the risks a company wants to sell, there is probably little reason not to use it, even if it gives inappropriate prices to other possible coverages. 